\title{
Performance Evaluation of Manual and Automated (MagNA Pure) Nucleic Acid Isolation in HPV Detection and Genotyping Using Roche Linear Array HPV Test
}

\author{
Aikaterini Chranioti, ${ }^{1}$ Evangelia Aga, ${ }^{1}$ Niki Margari, ${ }^{1}$ Christine Kottaridi, ${ }^{1}$ \\ Asimakis Pappas, ${ }^{2}$ Ioannis Panayiotides, ${ }^{3}$ and Petros Karakitsos ${ }^{1}$ \\ ${ }^{1}$ Department of Diagnostic Cytopathology, University General Hospital "Attikon”, Rimini 1, Chaidari, 12462 Athens, Greece \\ 23 rd Department of Obstetrics and Gynecology, University General Hospital "Attikon", Rimini 1, Chaidari, 12462 Athens, Greece \\ ${ }^{3}$ 2nd Department of Pathology, University General Hospital "Attikon", Rimini 1, Chaidari, 12462 Athens, Greece
}

Correspondence should be addressed to Petros Karakitsos, pkaraki@med.uoa.gr

Received 28 February 2011; Accepted 20 April 2011

Academic Editor: George Koliopoulos

Copyright (C) 2011 Aikaterini Chranioti et al. This is an open access article distributed under the Creative Commons Attribution License, which permits unrestricted use, distribution, and reproduction in any medium, provided the original work is properly cited.

\begin{abstract}
Nucleic acids of human papillomavirus (HPV) isolated by manual extraction method (AmpliLute) and automated MagNA pure system were compared and evaluated with cytohistological findings in 253 women. The concordance level between AmpliLute and MagNA was very good 93.3\% $(\kappa=0.864, P<.0001)$. Overall HPVpositivity detected by AmpliLute was 57.3\% $(30.4 \%$ as single and $27 \%$ as multiple infections) in contrast to MagNA $54.5 \%$ (32\% and 23\%, resp.). Discrepant results observed in 25 cases: $11 \mathrm{MagNA}(-) / \operatorname{AmpliLute}(+), 10$ of which had positive histology; $5 \mathrm{MagNA}(+) / \operatorname{AmpliLute}(-)$ with negative histology; 8 MagNA(+)/AmpliLute(+): in 7 of which AmpliLute detected extra HPV genotypes and 1 MagNA(invalid)/AmpliLute(+) with positive histology. Both methods performed well when compared against cytological (area under curve (AUC) of AmpliLute 0.712 versus 0.672 of MagNA) and histological diagnoses (AUC of AmpliLute 0.935 versus 0.877 of MagNA), with AmpliLute showing a slightly predominance over MagNA. However, higher sensitivities, specificities, and positive/negative predictive values were obtained by AmpliLute.
\end{abstract}

\section{Introduction}

It is now well established and widely accepted that virtually all cervical cancer and its immediate precancerous lesions arise from persisting cervical infection by some highly oncogenic HPV genotypes [1, 2]. The most important of these HPV genotypes are HPV16 and HPV18 which account for $\sim 70 \%$ of all invasive cervical cancers with minor variations in this percentage between continents [3].

Fifteen HPV genotypes have been to date classified as high-risk (HR) types $(16,18,31,33,35,39,45,51,52,56$, $58,59,68,73$, and 82$), 3$ as probably $\operatorname{HR}(26,53,66)$ and 12 as low-risk (LR) $(6,11,40,42,43,44,54,61,70,72,81$, and CP6108) $[1,4]$.

The majority of HPV infections are transient, but persistence of an HR HPV is a significant risk factor for the development of cervical cancer. This occurs only in a minority of infections and is an unpredicted event. It could be a genetic predisposition with an inadequate immune response and/or possible uncontrolled reaction with tumor suppressor genes $[5,6]$.

Type-specific detection of HPV is increasingly important for monitoring the impact of HPV vaccine implementation and as a tool for cervical cancer screening. As a consequence, standardization of laboratory methods for HPV detection and typing is important. The commercial HPV detection kits: Hybrid Capture II (Digene Corporation, Gaithersburg, Md, USA), Cervista HPV HR (Third Wave Technologies, Inc., Madison, USA) and Cervista 16/18 tests are approved by the FDA for use in routine screening of HPV. However, the above assays are unable to discriminate specific genotypes or to identify infections involving multiple genotypes and the Cervista assay detects only two HPV types (types 16 and 18). 
Various molecular assays for HPV detection and typing have been used in epidemiological studies, and they are based on two different technologies: (1) hybridization-based assays (e.g., HC II) and (2) PCR-based tests (e.g., GP5+/GP6+, PGMY09/11, INNO-LiPA HPV Genotyping (Innogenetics, Belgium), Linear Arrays HPV test (Roche Molecular Systems, Inc. Branchburg, NJ, USA), CLART HPV2 (Genomica, Madrid, Spain). The advantages and disadvantages of these two basically different methodologies have been extensively discussed [7-19].

The qualitative Linear Array HPV (LA-HPV) HPV genotyping test, developed by Roche Molecular Systems offers a reliable, sensitive, and standardized approach for HPV typing in cervical specimens. It is distributed as a research use only but it has been submitted for FDA review. This test utilizes amplification of target DNA by PCR and nucleic acid hybridization for the detection of 37 types in cervical cells collected into an LBC media. This test includes four steps: specimen preparation-DNA extraction, PCR amplification, hybridization of the amplified products with specific probes and colourimetric detection on the hybrids on strip [13, 17, 20-22]. Current specimen processing protocols recommend the use of manual extraction of DNA using the AmpliLute liquid media extraction kit, based on the QIAamp method (QIAGEN, Inc., Valencia, Calif, USA). An alternative method for DNA extraction is the automated MagNA Pure LC extraction system, developed by the same company.

The objective of this study was to evaluate and compare the automated MagNA pure DNA extraction method with the AmpliLute DNA extraction method in detecting HPV DNA form ThinPrep Pap tests using the linear array (LA) HPV genotyping and detection assays and also to correlate these results to cytological and histological diagnosis.

\section{Methods}

2.1. Clinical Specimens. In the present study, cervical brush specimens were obtained from women aged from 17 to 70 years who attended the gynecologic outpatient clinic of "Attikon" University Hospital, Athens, Greece, for opportunistic examination, between July 2009 and May 2010. Women considered eligible for the study if they fulfilled the following criteria: (a) they agreed to undergo colposcopy and if necessary cervical biopsy and (b) there was enough residual biological material, after cytological examination, for the two molecular assays to be completed. A total of 253 women met these criteria and were enrolled in the study. This patient population does not represent the general population of women attending public screening programs. Approval from the ethics committee was obtained before inclusion.

2.2. Cytological Diagnosis. Samples of ThinPrep Pap tests were collected by means of a Brun's-like brush. The PreservCyt vials (Corporate Headquarters: Hologic, Inc., Ltd., UK), containing the cell samples were addressed to the Department of Cytopathology of the aforementioned hospital for preparation of thin-layer slides using the ThinPrep 2000 Automated Slide Processor (Corporate Headquarters:
Hologic, Inc., Ltd., UK) according to the manufacturer's instructions. Cytological findings were interpreted according to the Bethesda classification system and were classified as follows: (a) within Normal Limits (WNL), (b) atypical squamous cells of undetermined significance (ASC-US), (c) low-grade squamous intraepithelial lesion (LSIL), and (d) high-grade squamous intraepithelial lesion (HSIL). The cytopathologists and the biologist conducting HPV testing were all blinded to the clinical profile to ensure unbiased reporting.

2.3. Histological Diagnosis. A cervical biopsy was performed if lesions were present upon colposcopy. All histological assessments were made blinded to the HPV DNA status of the participants. The histological evaluation revealed the following categories: negative HPV, CIN 1, CIN 2, and CIN 3. In case histology showed a CIN 2 or $\mathrm{CIN} 3$, the patient was referred for appropriate treatment.

2.4. DNA Extraction Methods. After slide preparation for cytological examination, the remaining PreservCyt samples were vortexed vigorously for $15 \mathrm{sec}$ to maximize homogeneity and two aliquots of $250 \mu \mathrm{L}$ and $1 \mathrm{~mL}$ were generated from each clinical specimen.

DNA was isolated using two different procedures (i) a $250 \mu \mathrm{L}$ aliquot was extracted by AmpliLute liquid media kit (Roche Molecular Systems) in conjunction with a QIAvac 24 plus vacuum system, according to the manufacturer's instructions in the product insert and (ii) a $1 \mathrm{~mL}$ aliquot was extracted by MagNA Pure LC extraction system using the DNA-I kit (blood cells high-performance protocol) (Roche Molecular Systems). Briefly, for the manual extraction, samples and HPV positive/negative controls were processed in parallel. Clinical samples were mixed by vortexing to form a homologous state, and $250 \mu \mathrm{L}$ were removed and lysed with proteinase $\mathrm{K}$ solution and buffer ATL at $56^{\circ} \mathrm{C}$ for $30 \mathrm{~min}$. The samples underwent a second incubation at $70^{\circ} \mathrm{C}$ for $15 \mathrm{~min}$ in the presence of buffer AL containing a carrier RNA. The lysate was then transferred to vacuum columns where isolation and purification of DNA was completed via washing of different solutions to bind DNA and remove other cellular materials. Extracted DNA was eluted into $120 \mu \mathrm{L}$ of buffer AVE. Specimens and controls were immediately stored at $2^{\circ} \mathrm{C}-8^{\circ} \mathrm{C}$ for up to 7 days or frozen at $-20^{\circ} \mathrm{C}$ for up to 8 weeks.

For automated extraction, the samples were prepared using a modified procedure involving the centrifugation of $1 \mathrm{~mL}$ aliquots of the PreservCyt samples at $13000 \times \mathrm{g}$ for $20 \mathrm{~min}$ prior to discarding of the supernatant. The resulted cell pellets were resuspended into $200 \mu \mathrm{L}$ of sterile phosphate-buffered saline, and the procedure of automated extraction was followed according to the manufacturer's instruction, using the DNA-I kit. The method is based on magnetic-bead technology with a special buffer containing chaotropic salts and proteinase K. Nucleic acids are bound to the surface of the magnetic glass particles. Cellular debris was removed by several washing steps, and the purified nucleic acids were eluted. $100 \mu \mathrm{L}$ in volume of extracted 
genomic DNA product was obtained, after the magnetic beads were separated from the solution. Specimens and controls were immediately stored at $2{ }^{\circ} \mathrm{C}-8^{\circ} \mathrm{C}$ for up to 7 days or frozen at $-20^{\circ} \mathrm{C}$ for up to 8 weeks. After nucleic acid purification all samples were analyzed by LA HPV assay for HPV genotyping.

2.5. LA-HPV Amplification (PCR). The LA genotyping test use a pool of biotinylated primers designed to amplify an approximately $450 \mathrm{bp}$ sequence within the polymorphic L1 region of the genome of the $37 \mathrm{HPV}$ genotypes. An additional 268 bp primer pair which targets the human $\beta$ globin gene is included in the assay to provide a control for cell adequacy, extraction, and amplification. PCR was carried out on each of the samples and controls, using the Linear Array HPV genotyping mastermix which contains: Tris buffer, potassium chloride, AmpliTaq, gold DNA polymerase (microbial), AmpErase, (uracil-N-glycosylate) enzyme (microbial), dATP, dCTP, dUTP, dGTP, dTTP, each of upstream and downstream primers (biotinylated) and $\beta$-globin primers, sodium azide, magnesium chloride, and amaranth dye. The reaction mixture contained $50 \mu \mathrm{L}$ of HPV mastermix and $50 \mu \mathrm{L}$ of eluted DNA. The amplification was performed on the Applied Biosystems Gold-plated 96-well GeneAmp PCR System 9700 (Applied Biosystems, Foster City, Calif, USA) using the following thermal profile: $2 \mathrm{~min}$ at $50^{\circ} \mathrm{C}, 9 \mathrm{~min}$ at $95^{\circ} \mathrm{C} ; 30 \mathrm{sec}$ at $95^{\circ} \mathrm{C}, 1 \mathrm{~min}$ at $55^{\circ} \mathrm{C}, 1 \mathrm{~min}$ at $72^{\circ} \mathrm{C}(40$ cycles) with a ramp rate set at $50 \%$ followed by $5 \mathrm{~min}$ at $72^{\circ} \mathrm{C}$ and a final hold at $72^{\circ} \mathrm{C}$ indefinitely. PCR amplicons were immediately denatured by the addition of $100 \mu \mathrm{L}$ of $(\mathrm{DN})$ denaturation reagent and stored at $4^{\circ} \mathrm{C}$ for further analysis within 7 days.

2.6. LA-HPV Detection. The detection of the HPV genotypes was carried out using the LA HPV detection kit. Once the amplification was completed, $75 \mu \mathrm{L}$ of denatured amplicon were added to the linear array strips that contain multiple copies of HPV genotype-specific probes in a defined area for all 37 genotypes and the $\beta$-globin reference lines. The HPV types detected are HPV6, 11, 16, 18, 26, 31, 33, $35,39,40,42,45,51-56,58,59,61,62,64,66-73,81-$ 84, IS39, and CP6108. The biotin-labeled amplicon was bound to the strips using a hybridization buffer in a shaking waterbath at $53^{\circ} \mathrm{C}$. Once bound, the strips were washed at high stringency to remove nonbound material and streptavidin-horseradish peroxidase conjugate was then added and bound to the biotin-labeled amplicon hybridized to the oligonucleotide probes. The strips were then washed with a substrate solution containing hydrogen peroxide and $3,3^{\prime}, 5,5^{\prime}$-tetramethylbenzidine (TMB). In the presence of hydrogen peroxide, the bound streptavidin-horseradish peroxidase catalyzed the oxidation of $\mathrm{TMB}$ to form a blue-colored complex, which precipitated at the probe positions where hybridization had occurred (colourimetric determination). This color precipitation allowed for manual reading of the strips and genotype detection by comparison with the HPV reference guide provided. LA test does not directly detect HPV52 but combines a set of probes that detects HPV33, -35 and -58 (HPV mix). Specimens that test negative for HPV33, -35 and -58 individually but are positive for HPV mix are considered to be HPV52 positive. The specimens that test positive for HPV mix and for HPV33, -35 and/or -58 have an uncertain HPV52 status, for this analysis, these specimens were considered to be HPV52 negative, since coinfection with HPV52 cannot be ruled out by this test.

The procedure performed into two physically separated areas (pre-PCR and post-PCR) in order to avoid contamination of samples with previously amplified products. All washes and hybridization steps were undertaken in a 24well tray with lid. The reading of the strips, produced by the two methods, was made by one well-experienced biomedical scientist. Discrepant interpretations were resolved by a second biomedical scientist and consensus review performed without knowledge of prior results. The LA-HPV test does not cross-react with a variety of viruses, bacteria, protozoa and yeast that could be present in cervical specimens.

2.7. Statistical Analysis. Pairwise comparison of AmpliLute method and MagNA pure method was performed by using kappa $(\kappa)$ statistics. A $\kappa$ value of 0 indicates no agreement better than chance, and $\kappa$ value of 1 indicates perfect agreement, $\kappa$ values from 0 to $0.20,0.21$ to $0.40,0.41$ to $0.60,0.61$ to 0.80 and 0.81 to 1.00 indicate poor, fair, moderate, good, and very good strengths of agreement, respectively. All $P$ values $<.001$ are considered statistically significant. Receiver operating characteristics (ROC) curve analysis was applied to calculate and compare AmpliLute method and MagNA pure method with cytological findings. In addition ROC analysis was applied to calculate and compare AmpliLute method and MagNA pure method with histological findings. All the statistical analyses were obtained with the statistical package for the social sciences (SPSS) computer software.

\section{Results}

A total of 253 women were analyzed in the present study by means of screening for the presence of HPV DNA by using two different DNA extraction methods. Out of the 253 cervical smears, 253 nucleic acid extracts were produced using the AmpliLute liquid media extraction method and 253 DNA extracts were generated using the MagNA pure automated extraction system. All women referred to colposcopy and if visible lesions were found, they were sampled. Patients with severe cervical diseases were further assessed.

The DNA extracts were evaluated by the LA-HPV genotyping test and compared against the reported cytological and histological diagnoses. The levels of sample adequacy for cytological examination and for nucleic acid extraction and amplification efficiency among the specimens, based on $\beta$-globin positivity, did not differ dramatically between the tests (Table 1). Sample adequacy was higher with AmpliLute extracts $(100 \%)$ than with the MagNA pure method $(99.6 \%)$ and cytology examination (97.6\%). Only one nucleic acid extract generated by the MagNA pure LC was invalid after LA 
TABLE 1: Adequacy of 253 samples extracted by the two methods tested by HPV LA test.

\begin{tabular}{lccc}
\hline \multirow{2}{*}{ Adequate sample } & \multicolumn{3}{c}{ No. of samples (\%) } \\
& Cytology & AmpliLute & MagNA pure* \\
\hline Yes & $247(97,6 \%)$ & $253(100 \%)$ & $252(99,6 \%)$ \\
No & $6(2,4 \%)$ & $0(0,0 \%)$ & $1(0,4 \%)$ \\
\hline Total & 253 & 253 & 253 \\
\hline
\end{tabular}

*DNA-I modified protocol using $1 \mathrm{~mL}$ PreservCyt sample.

genotyping test due to the absence of high and low $\beta$-globin result.

The comparison of HPV LA test results using AmpliLute extracts with equivalent MagNA pure extracts showed an overall concordance of $93.3 \%(\kappa=0.864, P<.001)$ (Table 2). HPV genotype profiles were identical in 228/253 (90.1\%) of specimens, including of $103 \mathrm{HPV}$ negative samples and 125 positive samples with identical HPV status detected by both methods. Out of 125 cases, the same HPV profile was identified in 73 single infections and in 52 multiple infections. Discrepant results observed in 25 samples including 11 cases which were AmpliLute(+)/MagNA(-), 5 cases AmpliLute $(-) / \mathrm{MagNA}(+), 5$ cases identified as single infections in MagNA but as multiple type infections in AmpliLute, 3 cases in which multiple infections were detected with different HPV profile between the two methods, and in one case, MagNA generated an invalid result as opposed to AmpliLute (Table 3).

Overall, 94/253 (37\%) women were diagnosed with normal cytology, 50/253 (19.8\%) were exhibited ASCUS, 91/253 (36\%) were diagnosed with LSIL, 5/253 (2\%) with LSIL but having some positions with HSIL, and 7/253 (3\%) with HSIL. In 6 cases (2.4\%) the cytological diagnosis was difficult due to inadequacy of the clinical samples (Tables 4 and 5).

HPV positivity detected by AmpliLute was slightly higher compared with MagNA Pure, $57.3 \%$ and 54.9\%, respectively. The largest percentage of samples negative for HPV DNA was found in WNL category. Analytically, for the cytological category WNL, HPV positivity observed by AmpliLute method was $29 / 94(31 \%)$, for ASCUS was $46 \%$, for LSIL $77 \%$, for LSIL/HSIL $100 \%$ and for HSIL 100\%. HPV positivity detected by the MagNA pure LC method for the aforementioned categories was: $33 \%, 44 \%, 78 \%, 80 \%$, and $100 \%$, respectively, (Tables 4 and 5).

Within the studied population, single and multiple type infections were present in every cytological diagnosis. In total, single HPV infections detected by the AmpliLute was $28.5 \%$ and by MagNA Pure $30.4 \%$ whereas multiple type of infection was observed in $26.5 \%$ and $23 \%$, respectively. In this study, multiple infections composed of up to five HPV genotypes in a plethora of combinations. The HPV distribution of single infections (divided into two categories according to the HPV genotype oncogenicity: LR and HR) as well as multiple infections (divided into three categories: LR (when only low-risk HPV types were present), HRLR (when low- and high-risk HPV types were present) and HR (when only high-risk HPV types were present) in all cytological categories studied are given in Figure 1

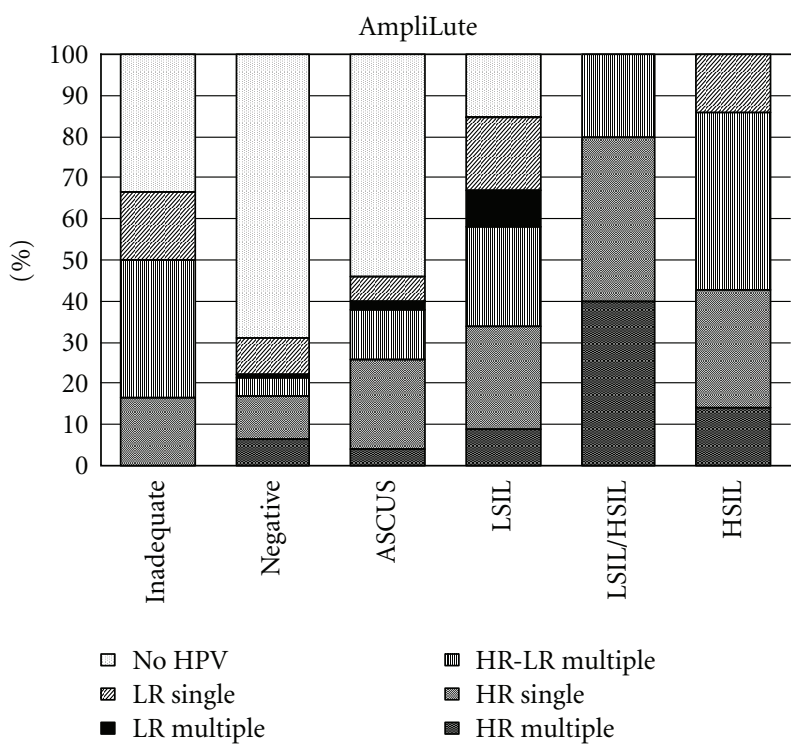

Figure 1: Prevalence of HR-HPV and LR-HPV types according to cytological diagnosis as detected by the AmpliLute extraction method.

for the AmpliLute method and in Figure 2 for the MagNA pure. More multiple infections were detected by AmpliLute method in all cytological categories compared with the MagNA pure ( $P$ not statistical significant). Analytically, for the cytological category WNL, the composition of multiple infections observed by AmpliLute method was at $6.4 \%$ with HR, $4.2 \%$ with HR-LR, and $1 \%$ with LR, for ASCUS at $4 \%$ with HR, $12 \%$ with HR-LR, and $2 \%$ with LR, for LSIL at 9\% with HR, 24\% with HR-LR, and 9\% with LR, for LSIL/HSIL at $40 \%$ with HR and $20 \%$ with HR-LR and for HSIL at 40\% with HR, 20\% with HR-LR. Multiple infections detected by the MagNA Pure LC method for the aforementioned categories was WNL at $4.2 \%$ with HR, $4.2 \%$ with HR-LR, and $2 \%$ with LR, for ASCUS at $4 \%$ with HR, $8 \%$ with HRLR and $2 \%$ with LR, for LSIL at $4 \%$ with HR, $23 \%$ with HR-LR and $9 \%$ with LR, for LSIL/HSIL at $20 \%$ with HR and $20 \%$ with HR-LR and for HSIL at $14 \%$ with HR, $43 \%$ with HR-LR. The HPV type prevalence according to the two extraction methods is given below with HPV16 being the most frequent type detected, in both types of infections and by the two methods, followed by HPV31, HPV53, HPV6, HPV33, HPV45, HPV42, and HPV51 as detected by the AmpliLute method and by HPV31, HPV53, HPV18, HPV51, HPV18, HPV6, and HPV33 as detected by the MagNA method (data not showed).

The two extraction methods were compared against the cytological findings (inadequate cytological samples were excluded). In terms of processing evaluation, AmpliLute method obtained better results than MagNA pure method (AmpliLute: sensitivity $(\mathrm{SE})=73.2 \%$, specificity $(\mathrm{SP})=$ $69.15 \%$, positive predictive value $(\mathrm{PPV})=79.43 \%$, negative predictive value $(\mathrm{NPV})=61.32 \%$; MagNA Pure: $\mathrm{SE}=$ $67.32 \%, \mathrm{SP}=67.02 \%, \mathrm{PPV}=76.87 \%, \mathrm{NPV}=55.75 \%)$. Both methods performed well when compared against the cytological diagnosis; nevertheless, the AmpliLute method 
TABle 2: Concordance between AmpliLute and MagNA Pure (DNA-I, $1 \mathrm{~mL}$ )* DNA extracts, in single and multiple HPV infections, identified by LA assay.

\begin{tabular}{|c|c|c|c|c|c|}
\hline \multicolumn{6}{|c|}{ No. of samples with the following LA results } \\
\hline \multirow[t]{2}{*}{ MagNA } & \multicolumn{5}{|c|}{ AmpliLute } \\
\hline & Single & Multiple (same) & Multiple (different) & Negative & Total \\
\hline Single HPV type & 73 & 5 & & 2 & 80 \\
\hline Multiple HPV type (same) & & 52 & & 3 & 55 \\
\hline Multiple HPV type (different) & & & 3 & & 3 \\
\hline Negative & 4 & 7 & & 103 & 114 \\
\hline Invalid & 1 & & & & 1 \\
\hline Total & 78 & 64 & 3 & 108 & 253 \\
\hline
\end{tabular}

TABLE 3: Analysis of cases with discrepant results.

\begin{tabular}{|c|c|c|c|}
\hline $\begin{array}{l}\text { Cytological } \\
\text { findings }\end{array}$ & $\begin{array}{l}\text { Histological } \\
\text { findings }\end{array}$ & AmpliLute & MagNA Pure \\
\hline \#1 WNL & HPV & $33,67,70$ & 67,70 \\
\hline \#2 WNL & $\mathrm{HPV}$ & 33,45 & $(-)$ \\
\hline \#3 WNL & No biopsy & $16,31,33,45$ & $(-)$ \\
\hline \#4 WNL & No biopsy & $(-)$ & 18 \\
\hline \#5 WNL & Negative & 33,45 & 45 \\
\hline \#6 WNL & No biopsy & $(-)$ & 6,58 \\
\hline \#7 WNL & No biopsy & $(-)$ & $16,33,35$ \\
\hline \#8 WNL & No biopsy & $(-)$ & 16,62 \\
\hline \#9 WNL & Negative & $31,33,42$ & 45 \\
\hline \#10 ASCUS & HPV & 35,53 & $35,53,54$ \\
\hline \#11 ASCUS & $\mathrm{HPV}$ & $39,42,52,84$ & 52 \\
\hline \#12 ASCUS & $\mathrm{HPV}$ & $6,16,52$ & 52 \\
\hline \#13 ASCUS & HPV & 16 & $(-)$ \\
\hline \#14 LSIL & $\mathrm{HPV}$ & 16 & $(-)$ \\
\hline \#15 LSIL & CIN 1 & $16,31,33,45$ & 16,31 \\
\hline \#16 LSIL & CIN 1 & $16,31,33,45$ & $(-)$ \\
\hline \#17 LSIL & CIN 1 & 42 & Invalid \\
\hline \#18 LSIL & CIN 1 & 31,16 & $(-)$ \\
\hline \#19 LSIL & Negative & $(-)$ & 59 \\
\hline \#20 LSIL & HPV & 16 & $(-)$ \\
\hline \#21 LSIL & CIN 1 & $6,16,33,45$ & $(-)$ \\
\hline \#22 LSIL & CIN 1 & 52,82 & 52 \\
\hline \#23 LSIL & CIN 1 & 16,31 & $(-)$ \\
\hline \#24 LSIL & HPV & 16 & $(-)$ \\
\hline \#25 LSIL/HSIL & $\mathrm{CIN} 2$ & $16,33,51$ & $(-)$ \\
\hline
\end{tabular}

demonstrated a slightest higher area under curve (AUC) 0.712 (Std. Error 0.035, 95\% CI: 0.644-0.779, $P<.001$ ) compared to AUC of MagNA pure method 0.672 (std. error 0.036, 95\% CI: 0.602-0.742, $P<.001)$.

The comparison of ThinPrep diagnosis and histological results is presented at Table 6 . Out of 253 colposcopic examinations, 145 women underwent biopsy. In 108 women, no visible lesions were found, and therefore, those women were not sampled. Positive histological result was found in 129 cases. From patients with normal cytology, only 13 out

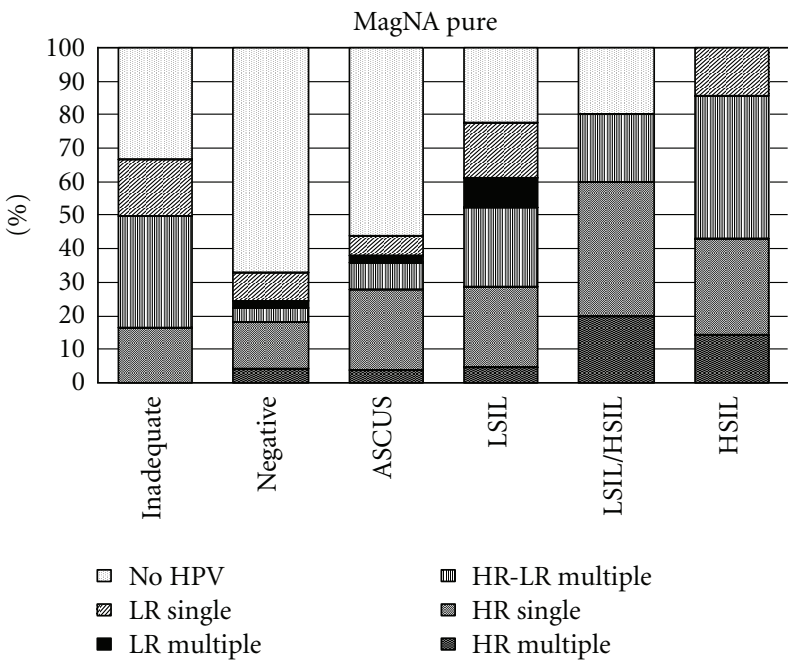

FIgURE 2: Prevalence of HR-HPV and LR-HPV types according to cytological diagnosis as detected by the MagNA pure extraction method.

of $94(13.8 \%)$ in biopsy had a HPV lesion or CIN 1 diagnosis. From patients with ASCUS, 14 out of 50 (28\%) had HPV in biopsy, $8 / 50$ (16\%) had CIN 1, whereas only 1 (2\%) patient had CIN 2. In LSIL, 29/91 (31.8\%) had a biopsy diagnosis of HPV, 39/91 (42.8\%) had CIN 1, 7/91 (7.7\%) had CIN 2, and only $2 / 91(2.2 \%)$ had CIN 3 . All cases classified as HSIL with ThinPrep cytology had a biopsy diagnosis of either CIN2 $(4 / 7,57.2 \%)$ or CIN3 (3/7, 42.8\%). In LSIL/HSIL category, 1 patient $(20 \%)$ had CIN1, $3 / 5(60 \%)$ had CIN2 and 1/5 (20\%) had CIN3. Four patients with no cytological diagnosis, due to inadequate of sampling, biopsy revealed lesions with HPV.

Results of biopsy reading and HPV genotyping by the two extraction methods are demonstrated in Tables 7 and 8 . Once more, AmpliLute method showed greater performance over MagNA pure (AmpliLute: $\mathrm{SE}=100 \%, \mathrm{SP}=87.5 \%, \mathrm{PPV}=$ $98.47 \%, \mathrm{NPV}=100 \%, \mathrm{FPR}=12.5 \%, \mathrm{FNR}=0.00 \%, \mathrm{OA}=$ 98.62\%, MagNA pure: $\mathrm{SE}=91.47 \%, \mathrm{SP}=81.25 \%, \mathrm{PPV}=$ $97.52 \%, \mathrm{NPV}=54.17 \%, \mathrm{FPR}=18.75 \%, \mathrm{FNR}=8.53 \%, \mathrm{OA}=$ 90.34\%). Comparison of AUC for AmpliLute method related to histological diagnosis was 0.935 (std. error $0.018,95 \%$ CI $(0.900-0.971), P<.001)$ and it was higher compared to 
TABLE 4: Distribution of HPV types (in single infections) detected by AmpliLute method against cytological diagnosis.

\begin{tabular}{|c|c|c|c|c|c|c|c|c|c|c|c|c|c|c|c|c|c|c|c|c|c|}
\hline \multirow[t]{2}{*}{ Cytology } & \multicolumn{21}{|c|}{ AmpliLute } \\
\hline & HPV Negative & 6 & 16 & 18 & 31 & 42 & 51 & 52 & 53 & 54 & 58 & 59 & 61 & 62 & 66 & 73 & 81 & 83 & 84 & Multiple & Total \\
\hline Negative & 65 & 2 & 7 & & 1 & & & & 1 & & & 2 & 3 & & & 1 & & 1 & & 11 & 94 \\
\hline ASCUS & 27 & & 6 & 1 & 2 & & & 2 & 2 & & & & & 1 & & & & & & 9 & 50 \\
\hline LSIL & 14 & 4 & 14 & 1 & 3 & 4 & 1 & 1 & 2 & 1 & 2 & & & & 1 & 1 & 1 & & 2 & 39 & 91 \\
\hline LSIL/HSIL & & & 2 & & & & & & & & & & & & & & & & & 3 & 5 \\
\hline HSIL & & & 1 & & 1 & & & & & & & & & & & 1 & & & & 4 & 7 \\
\hline Inadequate & 2 & & & 1 & & & & & & & & & & 1 & & & & & & 2 & 6 \\
\hline Total & 108 & 6 & 30 & 3 & 7 & 4 & 1 & 3 & 5 & 1 & 2 & 2 & 3 & 2 & 1 & 3 & 1 & 1 & 2 & 68 & 253 \\
\hline
\end{tabular}

TABLE 5: Distribution of HPV types (in single infections) detected by MagNA pure method against cytological diagnosis.

\begin{tabular}{|c|c|c|c|c|c|c|c|c|c|c|c|c|c|c|c|c|c|c|c|c|c|c|c|}
\hline \multirow[t]{2}{*}{ Cytology } & \multicolumn{23}{|c|}{ MagNA Pure } \\
\hline & $\begin{array}{c}\text { HPV } \\
\text { Negative }\end{array}$ & 6 & 16 & 18 & 31 & 42 & 45 & 51 & 52 & 53 & 54 & 58 & 59 & 61 & 62 & 66 & 73 & 81 & 83 & 84 & Multiple & Inadequate & Total \\
\hline Negative & 63 & 2 & 7 & 1 & 1 & & 2 & & & 1 & & & 2 & 3 & & & 1 & & 1 & & 10 & & 94 \\
\hline ASCUS & 28 & & 5 & 1 & 2 & & & & 4 & 2 & & & & & 1 & & & & & & 7 & & 50 \\
\hline LSIL & 20 & 4 & 11 & 2 & 3 & 3 & & 1 & 2 & 2 & 1 & 2 & 1 & & & 1 & 1 & 1 & & 2 & 33 & 1 & 91 \\
\hline LSIL/HSIL & 1 & & 2 & & & & & & & & & & & & & & & & & & 2 & & 5 \\
\hline HSIL & & & 1 & & 1 & & & & & & & & & & & & 1 & & & & 4 & & 7 \\
\hline Inadequate & 2 & & & 1 & & & & & & & & & & & 1 & & & & & & 2 & & 6 \\
\hline Total & 114 & 6 & 26 & 5 & 7 & 3 & 2 & 1 & 6 & 5 & 1 & 2 & 3 & 3 & 2 & 1 & 3 & 1 & 1 & 2 & 58 & 1 & 253 \\
\hline
\end{tabular}

AUC of MagNA pure method 0.877 (std. error $0.024,95 \%$ CI $(0.830-0.924), P<.001)$.

\section{Discussion}

Various research assays for HPV detection and typing have been used in epidemiological studies. The LA-HPV genotyping test provides a standardized, consistent and rapid means for HPV detection and genotyping. This test provides the capacity to identify 37 individual HPV genotypes within a given specimen and ascertain whether recurrent HPV positivity is, in fact, due to the persistence of a specific HR HPV genotype, meaning a substantially increased risk of disease progression [23-25]. Current specimen processing protocols recommend the use of manual extraction of DNA using the AmpliLute liquid media extraction kit, based on the QIAamp method (QIAGEN, Inc., Valencia, Calif, USA). This method of DNA preparation is time consuming and labor intensive and is prone to potential specimen cross-contamination, particularly when large numbers of specimens are being processed. An alternative method for DNA extraction is the automated MagNA pure LC extraction system, developed by the same company, which could facilitate the assay by minimizing the potential sample-contamination, handson time as well as increase labor efficiency and sample accuracy.

In the present study, we assessed DNA extracts form PreservCyt cervical samples, generated by the automated MagNA pure extraction system and by the manual AmpliLute method (both recommended by Roche) for HPV testing using the LA-HPV genotyping and detection assays. In addition, we correlated those results with the cytological and histological findings of the enrolled participants.

Among the 253 ThinPrep Pap tests analyzed in our study, only one extract from the MagNA pure modified DNA-I extraction protocol was found to be invalid due to the absence of low and high $\beta$-globin. In contrast, all nucleic acids generated from the AmpliLute protocol were valid for HPV DNA genotyping. This marginal difference in sample adequacy could be either due to the high AmpliLute protocol efficiency or to the variations in aliquoting the specific sample resulting in an inadequacy of cellular material for the automated procedure. Comparison of the HPV genotyping results, obtained with the AmpliLute DNA to those from the MagNA demonstrated a substantial level of agreement $(93.3 \%)$, with $\kappa$ value of 0.864 . Both extraction methods, in terms of qualitative results performed equally well when compared against the cytological diagnosis, with AmpliLute method demonstrating a small predominance (AUC of AmpliLute: 0.712 versus AUC of MagNA: 0.672). Nevertheless, the AmpliLute method exhibited higher sensitivity, specificity, positive and negative predictive values as opposed to MagNA method. The same outcome, AmpliLute method being more efficient than the MagNA, was noticed when we compared the two methods with the histological diagnosis. AUC of AmpliLute was 0.935 in contrast with the AUC of MagNA which was 0.877 .

HPV types identified in individual samples by each method are largely in agreement 90.1\% (228/253). In all studied cases, AmpliLute showed a slightly higher detection rate of HPV compared with MagNA. For the former, HPV 
TABLE 6: Correlation of cytological findings to histological diagnosis.

\begin{tabular}{|c|c|c|c|c|c|c|c|}
\hline \multirow[t]{2}{*}{ Cytology } & \multicolumn{7}{|c|}{ Histology } \\
\hline & No biopsy & Negative & HPV & CIN 1 & CIN 2 & CIN 3 & Total \\
\hline WNL & 79 & 2 & 4 & 9 & & & 94 \\
\hline ASCUS & 27 & & 14 & 8 & 1 & & 50 \\
\hline LGIL & & 14 & 29 & 39 & 7 & 2 & 91 \\
\hline HGIL & & & & & 4 & 3 & 7 \\
\hline LSIL/HSIL & & & & 1 & 3 & 1 & 5 \\
\hline Inadequate & 2 & & 4 & & & & 6 \\
\hline Total & 108 & 16 & 51 & 57 & 15 & 6 & 253 \\
\hline
\end{tabular}

TABLE 7: Distribution of HPV types (in single infections) detected by AmpliLute method against Histological Diagnosis.

\begin{tabular}{|c|c|c|c|c|c|c|c|c|c|c|c|c|c|c|c|c|c|c|c|c|c|}
\hline \multirow{2}{*}{ Histology } & \multicolumn{21}{|c|}{ AmpliLute } \\
\hline & HPV6 & 16 & 18 & 31 & 42 & 51 & 52 & 53 & 54 & 58 & 59 & 61 & 62 & 66 & 73 & 81 & 83 & 84 & Multiple & Negative & Total \\
\hline Negative & & & & & & & & & & & & & & & & & & & 2 & 14 & 16 \\
\hline HPV & 4 & 12 & 1 & & 3 & 1 & 1 & 4 & 1 & 2 & & & 2 & 1 & 1 & 1 & & 2 & 15 & & 51 \\
\hline CIN 1 & & 11 & 2 & 6 & 1 & & 2 & & & & & & & & & & & & 35 & & 57 \\
\hline CIN 2 & & 4 & & 1 & & & & & & & & & & & 1 & & & & 9 & & 15 \\
\hline CIN 3 & & 3 & & & & & & & & & & & & & & & & & 3 & & 6 \\
\hline No biopsy* & 2 & & & & & & & 1 & & & 2 & 3 & & & 1 & & 1 & & 4 & 94 & 108 \\
\hline Total & 6 & 30 & 3 & 7 & 4 & 1 & 3 & 5 & 1 & 2 & 2 & 3 & 2 & 1 & 3 & 1 & 1 & 2 & 68 & 108 & 253 \\
\hline
\end{tabular}

*Women without colposcopic findings.

overall positivity was calculated at $57.3 \%$ comprising $30.4 \%$ of HPV detected as single infection and $27 \%$ as multiple. And for the latter, the respective positivity was $54.5 \%, 32 \%$ as single and $23 \%$ as multiple type of infection. The prevalence of HPV-infected samples increased, in both methods, with the severity of cytological diagnoses: $30.8 \%$ of AmpliLute versus $33 \%$ of MagNA in the WNL, $46 \%$ versus $44 \%$ of ASCUS, $84.6 \%$ versus $77 \%$ of LSIL, $100 \%$ versus $80 \%$ of LSIL/HSIL and $100 \%$ by both of HSIL. Since, there are limited Greek epidemiological data available, studies that yielded similar findings in healthy women to our results, were the report by Papachristou et al. [26] who found that the corresponding prevalence was $31.5 \%$ and Agorastos et al. [27] at $36.3 \%$. Other studies in our country demonstrated that HPV DNA presence in WNL varied from $24 \%[28,29]$ to $18 \%[30,31]$ with the lowest prevalence reported at $2.5 \%$ [32]. This variability is also observed widely in the literature and is mainly due to the different criteria used for selecting the study population and also due to different molecular test applied. The biological meaning out of this is that latent HPV infections with no apparent underlying disease, which would otherwise not be diagnosed on cytological evaluation, are detectable with highly sensitive PCR-based methods.

AmpliLute correctly identified $87.5 \%$ of the negative histological cases as HPV negative samples compared to $81.2 \%$ of MagNA. In addition, in cases with histological evaluation from HPV up to CIN2, MagNA missed 10 cases counting for $8 \%(10 / 123)$ of the population with these specific histological abnormalities, whereas all those cases were accurately detected as HPV positive samples by AmpliLute. In 144 cases with cytological findings of WNL and ASCUS,
HPV was detected approximately in 52 cases (more than $50 \%$ of which were HR HPV types) and from which only 33 participants exhibited histological lesions of HPV up to CIN2. The remaining cases need to be followed up closely due to their elevated risk for developing a high-grade cervical lesion in the future.

The small number of cases investigated in this present study limits our ability to conclude correct and representative epidemiological data on HPV prevalence in Greek women. Nevertheless, data of this report on HPV distribution add to a rich body on literature demonstrating that HPV 16 was the most frequent type detected in both types of infections followed by HR HPV 31, 53, 33, 45, 18, and 51. The observation of HPV 53 being among the three most prevalent HR HPV types detected is consistent with findings of previous Greek studies [28, 31]. However, the prevalence and clinical role of HPV 51 needs to be clarified through further studies. Critical points on multiple infections are succinctly presented, since the detailed analysis of multiple infections identified in the clinical specimens was beyond the scope of this work and they will be discussed analytically on other report. Nevertheless, they were highly detected among the HPV positive participants: $47 \%(68 / 145)$ by the AmpliLute and 42\% (58/138) by the MagNA. Multiple type HPV infections were identified in approximately $50 \%$ of the HPV-infected individuals in WNL category, at 34\% in ASCUS, at 50\% in LSIL and in LSIL/HSIL and finally at $60 \%$ in HSIL category. The elevated incidence rate of multiple infections in our results are in line with the results described by Sandri et al. [10] who found multiple infections in $43 \%$ of the studied population and by Gargiulo et al. in $49.7 \%$ [33]. 
TABLE 8: Distribution of HPV types (in single infections) detected by MagNA Pure method against Histological Diagnosis.

\begin{tabular}{|c|c|c|c|c|c|c|c|c|c|c|c|c|c|c|c|c|c|c|c|c|c|c|c|}
\hline \multirow[t]{2}{*}{ Histology } & \multicolumn{23}{|c|}{ MagNA pure } \\
\hline & HPV6 & 16 & 18 & 31 & 42 & 45 & 51 & 52 & 53 & 54 & 58 & 59 & 61 & 62 & 66 & 73 & 81 & 83 & 84 & Inadequate & Multiple & Negative & Total \\
\hline Negative & & & & & & 2 & & & & & & 1 & & & & & & & & & & 13 & 16 \\
\hline HPV & 4 & 8 & 1 & & 3 & & 1 & 3 & 4 & 1 & 2 & & & 2 & 1 & 1 & 1 & & 2 & & 12 & 5 & 51 \\
\hline CIN 1 & & 11 & 3 & 6 & & & & 3 & & & & & & & & & & & & 1 & 29 & 4 & 57 \\
\hline CIN 2 & & 4 & & 1 & & & & & & & & & & & & 1 & & & & & 8 & 1 & 15 \\
\hline CIN 3 & & 3 & & & & & & & & & & & & & & & & & & & 3 & & 6 \\
\hline No biopsy* & 2 & & 1 & & & & & & 1 & & & 2 & 3 & & & 1 & & 1 & & & 6 & 91 & 108 \\
\hline Total & 6 & 26 & 5 & 7 & 3 & 2 & 1 & 6 & 5 & 1 & 2 & 3 & 3 & 2 & 1 & 3 & 1 & 1 & 2 & 1 & 58 & 114 & 253 \\
\hline
\end{tabular}

*Women without colposcopic findings.

Regarding the discordant results observed between the two extraction methods, as showed in Table 3, in $44 \%$ $(11 / 25)$ of the cases, MagNA failed in detecting HPV as opposed to the AmpliLute. Ten of these eleven cases were histological confirmed as $\geq \mathrm{HPV}$ and correctly identified by the AmpliLute. In $20 \%(5 / 25)$ of the cases, which were positive by the MagNA but negative by the AmpliLute method, there were either negative in histology or there were with normal cytology and without visible lesion upon colposcopy (thus for those women, cervical biopsy was not taken). In this regard, AmpliLute method gave a correct negative call and those cases could be considered as MagNA false positive results. In $32 \%(8 / 25)$ of cases, which were positive by the two methods but differing in the number of HPV genotypes detected, AmpliLute demonstrated higher level of detecting additional HPV genotypes in seven cases, apart from the common shared types, as opposed to MagNA. Those extra genotypes detected carry an increased clinical significance, since there were HR genotypes and could alter the clinical outcome of the patient. Only in one case, $4 \%$ (1/25) the HPV genotypes were completely different by the two methods and also in one case MagNA detected one extra genotype than the AmpliLute. The invalid result generated by the MagNA was HPV42 with CIN1 histology. Even though the patient population studied does not represent the general population attending our hospital, but only women who agreed to undergo further examination if necessary, the clinical samples tested covered a range of pathologies, from samples that were cytologically normal to samples that had HSIL. Therefore, the results (as well as the discordant result rate) for HPV detection generated by the two extraction methods demonstrated in the present study can be representative of the HPV infection in a screening population.

It is important to mention that the decision of utilizing the modified DNA protocol for the automated MagNA pure extraction system, was made based on a recent report. It compares DNA extraction efficiencies using the same extraction system with the incorporation of three different working DNA extraction kits: (i) blood cells high-performance protol (DNA-I kit), (ii) total nucleic acid (TNA) kit, and (iii) a modified DNA-I kit with the manual AmpliLute protocol for both AMPLICOR and LA HPV tests in 150 specimens [34]. Although the women enrolled in the above study had histological confirmed cervical abnormalities, no comparison was made between the DNA extracts and the
HPV genotyping test with their cytohistological findings. We used the modified DNA-I kit (blood cells high-performance protol) using $1 \mathrm{~mL}$ of PreservCyt sample as reported by Stevens et al. [34], since it performed better than the other two protocols and it was recommended by the author.

At this point, it is important to emphasise that even though for the manual AmpliLute method we used one fourth of biological material $(250 \mu \mathrm{L})$ as opposed to the automated MagNA DNA-I modified protocol $(1000 \mu \mathrm{L})$ and equal amount of DNA extract inputs were used for PCR amplification $(50 \mu \mathrm{L})$ and subsequently HPV detection, more HPV-positive cases were detected by the manual method. Someone would assume that increased HPV genotype detection would occur when a bigger amount of clinical sample is incorporated in the DNA extraction procedure, since more representative epithelial cells would be present in the sample tested, and thus increasing the possibility of HPV genotypes been detected by the assay used. The findings of our report, which are in contrast with previous reported one [34], declared the opposite, indicating that the current manual AmpliLute protocol for DNA preparation, provides adequate DNA quality, and consequently, it is capable of detecting HPV infections with high sensitivity. Having in mind that both methods gave comparative results when tested against cytology and histology, our data provide an additional advantage to AmpliLute, since reliable results can be obtained even when small volumes of biological material are available for molecular use.

In the literature, there is also a report that utilizing the same MagNA pure automated extraction system, compares the AMPLICOR HPV test to the INNO-LiPA HPV genotyping test, using only the TNA extraction kit for DNA isolation for AMPLICOR test [35], making, thus, difficult the direct comparison with this work. Several studies have undertaken assessment of the utility of various automated DNA extraction platforms in conjunction with the LA HPV test without comparing them with manual extraction methods $[34,36$, 37]. Moreover, in the literature, there are limited studies that address the variability in HPV genotyping introduced by small changes in front-end DNA extraction procedures prior to use in the LA HPV genotyping test $[38,39]$. From those reports, it was interesting found that minor changes to equally valid DNA extraction methods appeared to vary the assay's performance. For example, varying the volume of PreservCyt for DNA extraction or varying the centrifuge speed 
during DNA extraction or varying the amount of template DNA used for amplification can impact assay results. It is well documented and widely accepted that it is difficult to achieve reproducible and accurate HPV genotyping results using PCR-based methods, particularly when individual specimens may contain multiple concurrent infections and/or low viral copy numbers. Each of the many steps of testing, from collection of the cervical sample to the final recording of the result, can introduce important variability. Large-scale data comparing different methods of DNA isolation are needed to reach an optimal protocol for the HPV presence detection and accurate genotyping in order to monitor viral clearance, and most importantly HPV persistence, which is considered as a key factor in cervical cancer development. Moreover, accurate and sensitive methods for detection of HPV should be determined, since their performance can strongly affect the results of epidemiological studies and the clinical treatment strategy selected. Therefore, the MagNA extraction method should be tested against other automated and manual nucleic acids isolation techniques and in large population studies before being implemented and routinely used in laboratories. If would be proven accurate in detecting HPV infection, laboratories particularly those involved in large-scale HPV genotyping studies or handling a large amount of clinical specimens or can afford the cost of the automated procedure (more than two and a half times most pricey than the manual procedure) could profit from this automated nucleic acid isolation technique.

\section{Conclusion}

Accurate laboratory assays for the diagnosis of HPV infection are being recognized increasingly as essential for clinical management of women with cervical precancerous lesions. The first and most important step in molecular diagnosis of HPV infection is the nucleic acid isolation. An alternative approach to manual extraction procedures, which are time consuming and labor intense, is the automated processing of clinical specimens for HPV detection and genotyping which minimizes the potential sample contamination and the hands-on time. From our data, it was concluded that both DNA extraction methods demonstrated similar clinical performance, with no significant difference for any of the outcomes assessed even if for some outcomes the AmpliLute method exhibited higher sensitivity, specificity positive and negative predictive values as opposed to MagNA methods. Based on the results of this study, the automated nucleic acid isolation method should be tested versus other automated and manual techniques before it is routinely implemented. In addition, additional studies with larger populations are required to be carried out using the automated extraction system in order for its potential value to accurate HPV detection been determined.

\section{Authors Contribution}

A. Chranioti and P. Karakitsos conceived and coordinated the study; E. Age and C. Kottaridi performed the molecular analysis; N. Margari performed the statistical analysis; A. Pappas collected thin prep samples and performed colposcopy/biopsy; I. Panayiotides performed the pathological evaluation; P. Karakitsos performed the thin prep evaluation; A. Chranioti wrote the paper.

\section{Conflict of Interests}

The authors declare there is no conflict of interests.

\section{References}

[1] N. Muñoz, F. X. Bosch, and S. De Sanjosé, "Epidemiologic classification of human papillomavirus types associated with cervical cancer," New England Journal of Medicine, vol. 348, no. 6, pp. 518-527, 2003.

[2] J. M. M. Walboomers, M. V. Jacobs, and M. M. Manos, "Human papillomavirus is a necessary cause of invasive cervical cancer worldwide," Journal of Pathology, vol. 189, no. 1, pp. 12-19, 1999.

[3] J. S. Smith, L. Lindsay, and B. Hoots, "Human papillomavirus type distribution in invasive cervical cancer and high-grade cervical lesions: a meta-analysis update," International Journal of Cancer, vol. 121, no. 3, pp. 621-632, 2007.

[4] E. Beerens, L. Van Renterghem, and M. Praet, "Human papillomavirus DNA detection in women with primary abnormal cytology of the cervix: prevalence and distribution of HPV genotypes," Cytopathology, vol. 16, no. 4, pp. 199-205, 2005.

[5] K. S. Cuschieri, H. A. Cubie, and M. W. Whitley, "Persistent high risk HPV infection associated with development of cervical neoplasia in a prospective population study," Journal of Clinical Pathology, vol. 58, no. 9, pp. 946-950, 2005.

[6] P. Londesborough, H. O. Linda, G. Terry, J. Cuzick, C. Wheeler, and A. Singer, "Human papillomavirus genotype as a predictor of persistence and development of highgrade lesions in women with minor cervical abnormalities," International Journal of Cancer, vol. 69, no. 5, pp. 364-368, 1996.

[7] J. Monsonego, G. Pollini, and M. J. Evrard, "Linear array genotyping and hybrid capture II assay in detecting human papillomavirus genotypes in women referred for colposcopy due to abnormal Papanicolaou smear," International Journal of STD and AIDS, vol. 19, no. 6, pp. 385-392, 2008.

[8] J. Monsonego, F. X. Bosch, and P. Coursaget, "Cervical cancer control, priorities and new directions," International Journal of Cancer, vol. 108, no. 3, pp. 329-333, 2004.

[9] J. Monsonego, J. M. Bohbot, and G. Pollini, "Performance of the roche AMPLICOR ${ }^{\circledR}$ human papillomavirus (HPV) test in prediction of cervical intraepithelial neoplasia (CIN) in women with abnormal PAP smear," Gynecologic Oncology, vol. 99, no. 1, pp. 160-168, 2005.

[10] M. T. Sandri, D. Riggio, and M. Salvatici, "Typing of human papillomavirus in women with cervical lesions: prevalence and distribution of different genotypes," Journal of Medical Virology, vol. 81, no. 2, pp. 271-277, 2009.

[11] P. E. Castle, P. E. Gravitt, D. Solomon, C. M. Wheeler, and M. Schiffman, "Comparison of linear array and line blot assay for detection of human papillomavirus and diagnosis of cervical precancer and cancer in the atypical squamous cell of undetermined significance and low-grade squamous intraepithelial 
lesion triage study," Journal of Clinical Microbiology, vol. 46, no. 1, pp. 109-117, 2008.

[12] Y. L. Woo, I. Damay, M. Stanley, R. Crawford, and J. Sterling, "The use of HPV Linear Array Assay for multiple HPV typing on archival frozen tissue and DNA specimens," Journal of Virological Methods, vol. 142, no. 1-2, pp. 226-230, 2007.

[13] P. E. Gravitt, C. L. Peyton, R. J. Apple, and C. M. Wheeler, "Genotyping of 27 human papillomavirus types by using L1 consensus PCR products by a single-hybridization, reverse line blot detection method," Journal of Clinical Microbiology, vol. 36, no. 10, pp. 3020-3027, 1998.

[14] C. Y. Lin, H. C. Chen, and R. W. Lin, "Quality assurance of genotyping array for detection and typing of human papillomavirus," Journal of Virological Methods, vol. 147, no. 1, pp. 194-195, 2008.

[15] C. Perrons, B. Kleter, R. Jelley, H. Jalal, W. Quint, and R. Tedder, "Detection and genotyping of human papillomavirus DNA by SPF10 and MY09/11 primers in cervical cells taken from women attending a colposcopy clinic," Journal of Medical Virology, vol. 67, no. 2, pp. 246-252, 2002.

[16] M. Schiffman, R. Herrero, and R. Desalle, "The carcinogenicity of human papillomavirus types reflects viral evolution," Virology, vol. 143, no. 1, pp. 45-54, 2007.

[17] M. P. Stevens, E. Rudland, S. M. Garland, and S. N. Tabrizi, "Assessment of MagNA pure LC extraction system for detection of human papillomavirus (HPV) DNA in PreservCyt ${ }^{\circledR}$ samples by the Roche AMPLICOR and LINEAR ARRAY HPV Tests," Journal of Clinical Microbiology, vol. 44, no. 7, pp. 24282433, 2006.

[18] B. J. LaMere, J. Kornegay, B. Fetterman, M. Sadorra, J. Shieh, and P. E. Castle, "Human papillomavirus genotyping after denaturation of specimens for Hybrid Capture 2 testing: feasibility study for the HPV persistence and progression cohort," Journal of Virological Methods, vol. 146, no. 1-2, pp. 80-85, 2007.

[19] S. P. Day, A. Hudson, and T. Mast, "Analitical performance of the investigational use only Cervista HPV HR test as determined by a multi-center study," Journal of Clinical Virology, vol. 45, supplement 1, pp. 63-72, 2009.

[20] D. Van Hamont, M. A. P. C. Van Ham, J. M. J. E. Bakkers, L. F. A. G. Massuger, and W. J. G. Melchers, "Evaluation of the SPF10-INNO LiPA human papillomavirus (HPV) genotyping test and the Roche Linear Array HPV genotyping test," Journal of Clinical Microbiology, vol. 44, no. 9, pp. 3122-3129, 2006.

[21] P. E. Castle, M. Sadorra, F. Garcia, E. B. Holladay, and J. Kornegay, "Pilot study of a commercialized human papillomavirus (HPV) genotyping assay: comparison of HPV risk group to cytology and histology," Journal of Clinical Microbiology, vol. 44, no. 11, pp. 3915-3917, 2006.

[22] F. Coutlée, D. Rouleau, and P. Petignat, "Enhanced detection and typing of human papillomavirus (HPV) DNA in anogenital samples with PGMY primers and the Linear Array HPV genotyping test," Journal of Clinical Microbiology, vol. 44, pp. 1998-2006, 2006.

[23] K. S. Cuschieri, M. J. Whitley, and H. A. Cubie, "Human papillomavirus type specific DNA and RNA persistenceimplications for cervical disease progression and monitoring," Journal of Medical Virology, vol. 73, no. 1, pp. 65-70, 2004.

[24] G. Y. F. Ho, R. D. Burk, and S. Klein, "Persistent genital human papillomavirus infection as a risk factor for persistent cervical dysplasia," Journal of the National Cancer Institute, vol. 87, no. 18, pp. 1365-1371, 1995.
[25] K. L. Wallin, F. Wiklund, and T. Angström, "Type-specific persistance of human papillomavirus DNA before the development of invasive cervical cancer," The New England Journal of Medicine, vol. 341, pp. 1633-1638, 1999.

[26] E. Papachristou, V. Sypsa, and D. Paraskevis, "Prevalence of different HPV types and estimation of prognostic risk factors based on the linear array HPV genotyping test," Journal of Medical Virology, vol. 81, no. 12, pp. 2059-2065, 2009.

[27] T. Agorastos, J. Bontis, and A. F. Lambropoulos, "Epidemiology of human papillomavirus infection in Greek asymptomatic women," European Journal of Cancer Prevention, vol. 4, no. 2, pp. 159-167, 1995.

[28] C. Kroupis, G. Thomopoulou, T. G. Papathomas, N. Vourlidis, and A. C. Lazaris, "Population-based study of human papillomavirus infection and cervical neoplasia in Athens, Greece," Epidemiology and Infection, vol. 135, no. 6, pp. 943-950, 2007.

[29] V. Labropoulou, E. Diakomanolis, S. Dailianas, K. Kalpaktsoglou, A. Balamotis, and P. Mavromara, "Type-specific prevalence of genital human papillomaviruses in benign, premalignant, and malignant biopsies in patients from Greece," Sexually Transmitted Diseases, vol. 24, no. 8, pp. 469-474, 1997.

[30] S. Tsiodras, J. Georgoulakis, and A. Chranioti, "Hybrid capture vs. PCR screening of cervical human papilloma virus infections. Cytological and histological associations in 1270 women," BMC Cancer, vol. 10, article 53, 2010.

[31] P. Stamataki, A. Papazafiropoulou, and I. Elefsiniotis, "Prevalence of HPV infection among Greek women attending a gynecological outpatient clinic," BMC Infectious Diseases, vol. 10 , article 27, 2010.

[32] T. Agorastos, K. Dinas, and B. Lloveras, "Cervical human papillomavirus infection in women attending gynaecological outpatient clinics in northern Greece," European Journal of Cancer Prevention, vol. 13, no. 2, pp. 145-147, 2004.

[33] F. Gargiulo, M. A. De Francesco, and C. Schreiber, "Prevalence and distribution of single and multiple HPV infections in cytologically abnormal cervical samples from Italian women," Virus Research, vol. 125, no. 2, pp. 176-182, 2007.

[34] M. P. Stevens, E. Rudland, S. M. Garland, and S. N. Tabrizi, "Assessment of MagNA pure LC extraction system for detection of human papillomavirus (HPV) DNA in PreservCyt samples by the Roche AMPLICOR and LINEAR ARRAY HPV Tests," Journal of Clinical Microbiology, vol. 44, no. 7, pp. 24282433, 2006.

[35] M. A. P. C. van Ham, J. M. J. E. Bakkers, G. K. Harbers, W. G. V. Quint, L. F. A. G. Massuger, and W. J. G. Melchers, "Comparison of two commercial assays for detection of human papillomavirus (HPV) in cervical scrape specimens: validation of the Roche AMPLICOR HPV test as a means to screen for HPV genotypes associated with a higher risk of cervical disorders," Journal of Clinical Microbiology, vol. 43, no. 6, pp. 2662-2667, 2005.

[36] P. E. Castle, M. Sadorra, F. Garcia, E. B. Holladay, and J. Kornegay, "Pilot study of a commercialized human papillomavirus (HPV) genotyping assay: comparison of HPV risk group to cytology and histology," Journal of Clinical Microbiology, vol. 44, no. 11, pp. 3915-3917, 2006.

[37] S. Dodson, T. Kerr, R. A. Hubbard, and N. T. Potter, "Evaluation of the Qiagen BioRobot MDx and the Roche Amplicor HPV microwell Plate research reagents and the Roche Linear Array HPV Test (RUO) for the extraction and detection of HPV DNA from ThinPrep ${ }^{\circledR}$ specimens," The Journal of Molecular Diagnostics, vol. 7, p. 670, 2005. 
[38] G. D’Souza, E. Sugar, W. Ruby, P. Gravitt, and M. Gillison, "Analysis of the effect of DNA purification on detection of human papillomavirus in oral rinse samples by PCR," Journal of Clinical Microbiology, vol. 43, no. 11, pp. 5526-5535, 2005.

[39] S. T. Dunn, R. A. Allen, S. Wang, J. Walker, and M. Schiffman, "DNA extraction: an understudied and important aspect of HPV genotyping using PCR-based methods," Journal of Virological Methods, vol. 143, no. 1, pp. 45-54, 2007. 


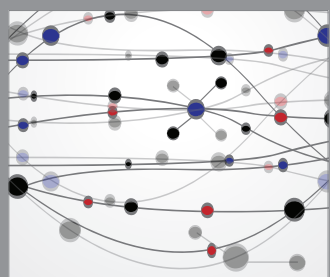

The Scientific World Journal
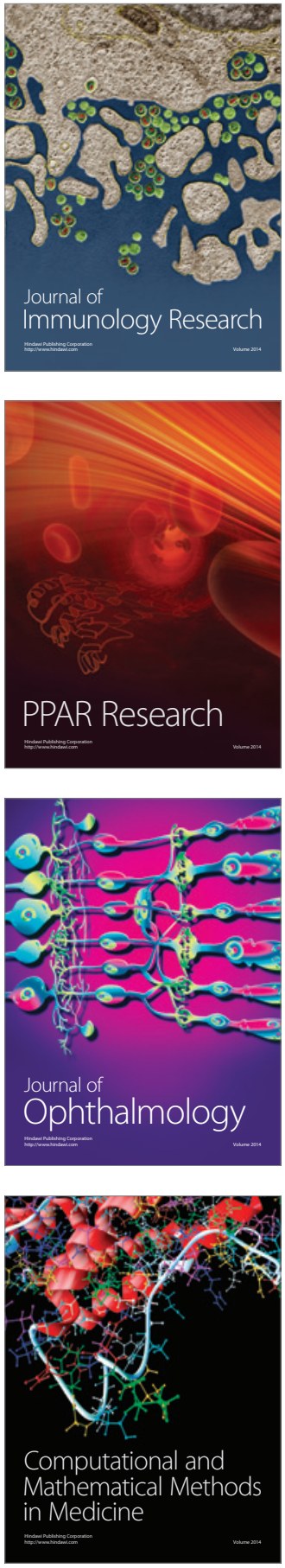

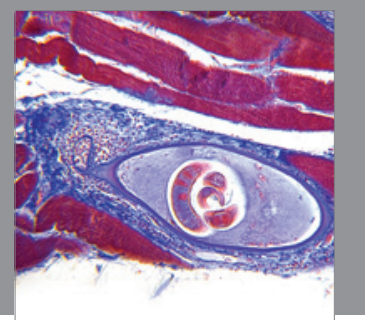

Gastroenterology

Research and Practice
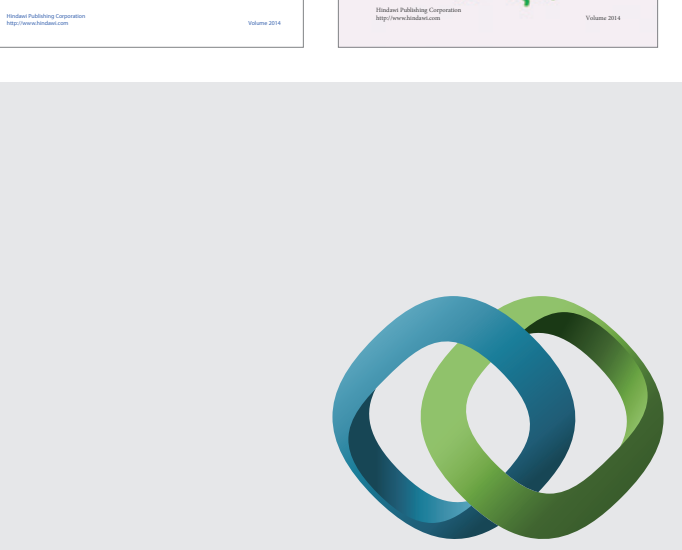

\section{Hindawi}

Submit your manuscripts at

http://www.hindawi.com
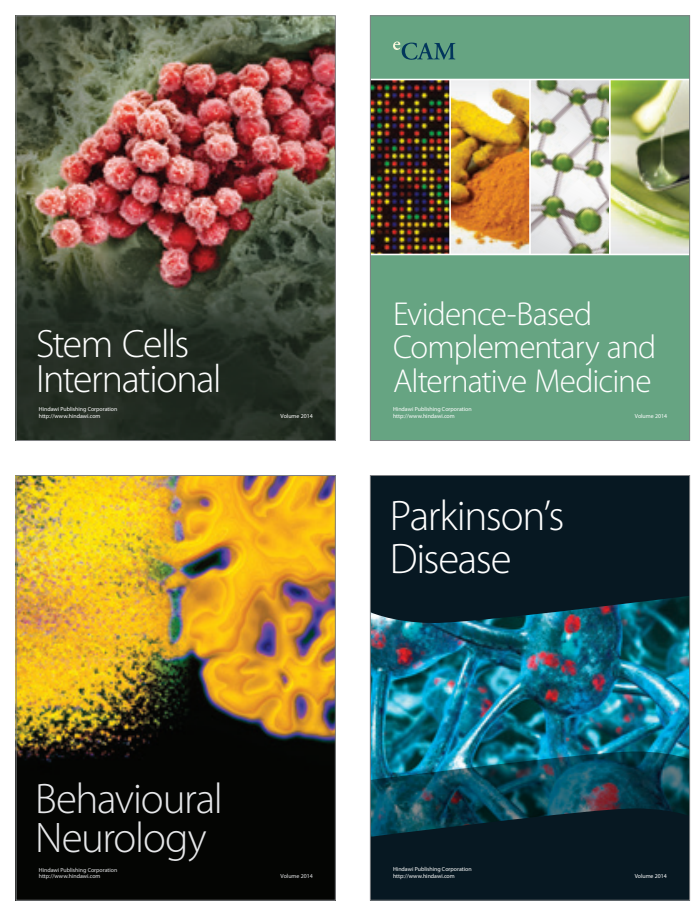

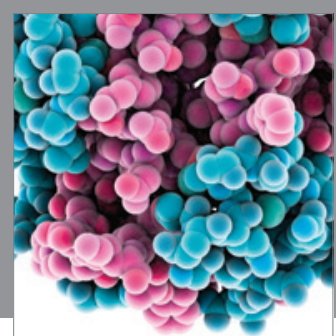

Journal of
Diabetes Research

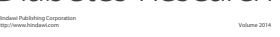

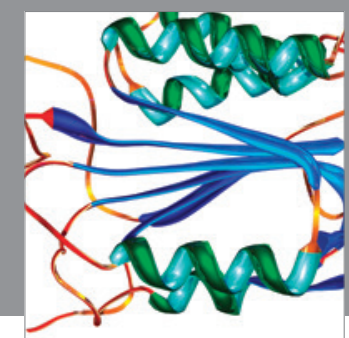

Disease Markers
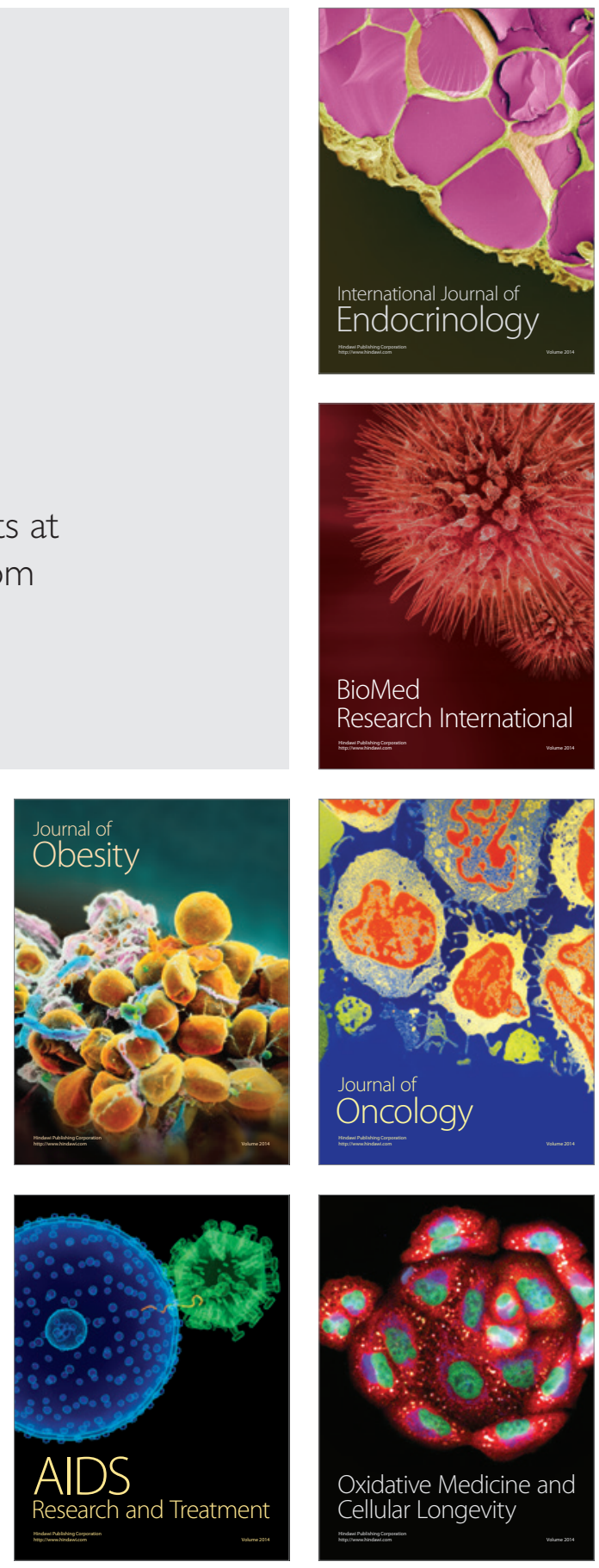